\title{
PRE-TREATMENT FACTORS RELATED TO COGNITIVE FUNCTIONING IN WOMEN NEWLY DIAGNOSED WITH BREAST CANCER
}

\author{
BERNADINE CIMPRICH ${ }^{\mathrm{a}, *}$, HEEYOUNG SO ${ }^{\mathrm{b}}$, DAVID L. RONIS ${ }^{\mathrm{c}}$ and CHRISTINE TRASK ${ }^{\mathrm{d}}$ \\ ${ }^{\mathrm{a}}$ University of Michigan, USA \\ ${ }^{\mathrm{b}}$ Chungnam National University Daejon, South Korea \\ ${ }^{\mathrm{c}}$ Department of Veterans Affairs, University of Michigan, Ann Arbor, MI, USA \\ ${ }^{\mathrm{d}}$ NeuroBehavioral Resources, Inc., Ann Arbor, MI, USA
}

\begin{abstract}
SUMMARY
Women treated for breast cancer have shown cognitive deficits with reduced capacity to focus and concentrate or to direct attention. This study examined the relationship between cognitive function prior to any treatment for breast cancer and individual factors including age, education, menopausal status, chronic health problems, and distress. Women newly diagnosed with breast cancer $(N=184)$, ages $27-86$ years, were assessed with standardized attention tests, self-reports of effectiveness in cognitive functioning, and measures of distress at about 18 days before surgery. Measured performance on the cognitive tests was not significantly correlated to self-reports of effectiveness in cognitive functioning. Age, education, presence of a chronic health problem, and menopausal status, but not distress, were associated with performance on the cognitive tests. Only age and education, however, were significant $(p<0.001)$ predictors of overall performance on the cognitive tests, when controlling covariates. In contrast, symptom and mood distress significantly $(p<0.001)$ predicted perceptions of effectiveness in cognitive functioning. Thus, different factors were associated with measured performance versus self-reports of cognitive functioning. Individual factors that predispose to lowered effectiveness in cognitive functioning prior to treatment in women newly diagnosed with breast cancer are discussed. Copyright (C) 2004 John Wiley \& Sons, Ltd.
\end{abstract}

\section{INTRODUCTION}

A growing number of studies have documented the presence of cognitive deficits in women treated for breast cancer. A common finding across studies is the presence of problems of attention and concentration of varying severity during and following breast cancer treatment (Ahles and Saykin, 2001; Cimprich and Ronis, 2001). The capacity to focus and concentrate, or to direct attention, is essential for effective cognitive functioning. A strong Capacity to Direct Attention (CDA) is needed to acquire important information, to make decisions, and to carry out self-care activities. Increasing understanding of problems of attention is of key

\footnotetext{
*Correspondence to: School of Nursing, University of Michigan, 400 N. Ingalls, Ann Arbor, MI 48109-0482, USA. E-mail: cimprich@umich.edu (B. Cimprich)
}

importance in individuals treated for cancer, since this cognitive capacity supports higher-level cognitive functions (Posner and Dehaene, 1994; Smith and Jonides, 1999). Little research has been done to determine what factors might predispose women newly diagnosed with breast cancer to impairments of attention. Thus, the purpose of this study was to examine possible factors that might be associated with the capacity to direct attention prior to any treatment in women newly diagnosed with breast cancer.

Directed attention refers to the cognitive ability to actively block or inhibit a competing stimulus in purposeful or goal-directed activity (James, 1983; Posner and Dehaene, 1994; Posner, 1995). In particular, the capacity to direct attention permits purposeful mental activity through inhibition of distractions or competing information in the environment (internal or external) (James, 1983; Kaplan and Kaplan, 1982; Posner and Dehaene, 
1994; Posner, 1995). This form of selective attention is also known as voluntary or controlled attention (James, 1983; Kaplan and Kaplan, 1982; Posner and Dehaene, 1994; Posner, 1995). Directed attention provides neural inhibitory control for key aspects of cognitive function, including perception and working memory (Posner and Dehaene, 1994; Posner, 1995) and supports higher-level executive functions such as acquiring information, planning and carrying out effortful tasks, and regulating behavior to meet desired goals (Smith and Jonides, 1999; Lezak, 1995). Thus, the CDA is of fundamental concern when considering possible cognitive deficits in individuals with cancer, since even a small loss in attentional capacity can interfere with mental clarity and higher-level cognitive activities.

The presence of cognitive deficits, including subtle and more severe problems of attention, has been documented during and following chemotherapy for breast cancer (for comprehensive reviews see Ahles and Saykin, 2001; Phillips and Bernhard, 2003). The reported incidence of cognitive deficits following chemotherapy for breast cancer ranges from $17 \%$ to $35 \%$ (Ahles and Saykin, 2001). Fatigue-related losses in CDA also have been documented following breast cancer surgery (Cimprich, 1992, 1993, 1999). In most instances the research has been done following the initiation of cancer treatment. Although certain individual factors, such as age, education, menopausal status, and psychological distress can affect CDA, little research has been done to determine to what extent such factors influence attention in women diagnosed with breast cancer prior to any treatment.

Aging has been associated with a loss of CDA in healthy, cognitively intact, older men and women in a large number of studies (e.g. Braver et al., 2001; Allen et al., 2001; Ylikoski et al., 1998; Zacks and Hasher, 1994), indicating a loss of efficiency in directing attention in a demanding or distracting environment, as would be expected when dealing with a diagnosis of breast cancer. In this regard, older women, 55-79 years of age, newly diagnosed with breast cancer showed significantly lower CDA prior to any treatment than women similar in age without breast cancer (Cimprich and Ronis, 2001).

A key aspect of physiological aging in women is the reduction in reproductive hormones with normal menopause. Reductions in estrogen and progesterone in post-menopausal women have been associated with cognitive changes, involving a decline in attention, learning and memory; however, it has not been determined how menopausal status might affect cognitive function in women newly diagnosed with breast cancer (Bender et al., 2001).

Education has been shown to affect performance scores over a wide number of cognitive measures in healthy individuals (Lezak, 1995; Leckliter and Matarazzo, 1989; Capitani et al., 1996). In general, higher levels of education have been associated with better performance on measures of attention, particularly on verbal measures and more complex or effortful tasks requiring directed attention (Lezak, 1995).

Mood distress, including depressive symptoms and anxiety, has been more generally associated with self-reports (perceptions) of poorer cognitive functioning in individuals with cancer. Self-reports of attention and memory problems in 91 diseasefree, adult survivors of lymphoma were related to higher affective distress scores including anxiety and depression, but not to actual performance on objective neuropsychological measures (Cull et al., 1996). Similarly, following standard or high dose chemotherapy for breast cancer, women's subjective reports of problems with attention and memory were related to psychological distress but not to actual performance on the objective test measures (van Dam et al., 1998). Finally, a study of 74 women newly diagnosed with breast cancer also showed that higher mood distress scores in the pre-treatment period were significantly correlated with perceptions of lowered effectiveness in cognitive functioning (Cimprich, 1999). Taken together, these findings suggest a more global relationship between affective distress and perceptions of lowered effectiveness in cognitive functioning (and vice versa) in individuals with cancer that appears to be unrelated to cognitive performance on neuropsychological testing.

In summary, previous studies in healthy populations and individuals with cancer suggest a number of factors that might interfere with CDA. However, there has been no previous research to determine whether or to what extent such factors might affect attention and cognitive functioning prior to any treatment in women newly diagnosed with cancer. Thus, the purposes of this study were to determine: (a) the relationship of CDA with pertinent demographic and medical characteristics, that is, age, menopausal status, education and presence of health problems, prior to any 
treatment in women newly diagnosed with breast cancer; (b) the relationship of CDA with pretreatment symptom distress and mood state; and (c) the combined value of demographic and medical characteristics and distress factors in predicting CDA prior to any breast cancer treatment.

\section{METHOD}

\section{Participants}

The study participants were 184 female volunteers drawn from the population of patients treated for breast cancer at a university medical center with a comprehensive cancer treatment program. Only patients with early stage (Stages 0 , I, or II) breast cancer were eligible for the study. Individuals were excluded for pre-existing conditions that could influence CDA or performance of measures, such as documented cognitive, mental, or affective disorders, prescribed medication known to enhance or impair attention, and insufficient command of the English language.

A description of demographic and medical characteristics is shown in Table 1. The women ranged in age from 27 to 86 years and were relatively well educated, with about $76 \%$ having at least some college education. The majority of participants were married, and $90 \%$ were white. Half of the respondents $(50 \%)$ reported having some other chronic health problem such as hypertension, diabetes or heart disease. The majority of the women were post-menopausal and were diagnosed with Stage I breast cancer. None of the women in the study reported being on hormone replacement therapy at the time of testing.

\section{MEASURES}

The measures of CDA, or the ability to inhibit a competing stimulus while focusing on a task, were Digit Span and the Trail Making test. Both tests are theoretically congruent requiring inhibition of a competing stimulus to perform the task accurately. In addition, a measure of short-term memory, Three Shapes and Three Words, was included since there is a close functional connection between attentional control and short-term
Table 1. Demographic and medical characteristics of sample $(N=184)$

\begin{tabular}{ll}
\hline Characteristics & \\
\hline Age & \\
Mean (SEM) & $54.6(0.84)$ \\
(Min-Max) & $(27-86)$ \\
& $N(\%)$ \\
Education & \\
High school or less & $32(21)$ \\
College (full/some) & $107(54)$ \\
Graduate degree & $40(22)$ \\
Not reported & $5(3)$ \\
Race & \\
White & $165(90)$ \\
Non-white & $19(10)$ \\
Marital status & \\
Married & $117(64)$ \\
Single & $67(36)$ \\
Other chronic health problems & \\
Yes & $92(50)$ \\
No & $92(50)$ \\
Menopausal state & \\
Pre- & $51(28)$ \\
Peri & $13(7)$ \\
Post- & $120(65)$ \\
Stage of disease & \\
0 & $17(9)$ \\
I & $97(53)$ \\
II & $63(34)$ \\
Not reported & $7(4)$ \\
\hline
\end{tabular}

${ }^{\mathrm{a}}$ Single $=$ Widowed, divorced, separated, never married.

memory, with working memory especially vulnerable to loss of inhibitory control. Perceptions of effectiveness in activities requiring directed attention was measured with a self-rating scale, the Attentional Function Index.

Digit Span: This standardized test of attention consists of two separate measures, Digit Span Forward (DSF) and Backward (DSB) (Lezak, 1995). DSF measures the bits of information that a person can attend to at one time in a task requiring repetition of a random series of digits presented at a rate of one digit per second. DSB, the more rigorous test, requires sustained directed attention to mentally track and reverse a random series of digits. The scores were the number of digits repeated correctly in forward (DSF) and backward (DSB) sequences before two failed trials.

Trail Making Test (TMT) (Reitan, 1958): The TMT is a standard test of attention (Lezak, 1995) requiring effective inhibition of competing responses 
to correctly complete the task in a timely manner. The TMT consists of two parts. In part A, the participant is required to connect a series of randomly placed circles numbered $1-25$ in correct order as quickly as possible. Part B, the higher demand condition, requires the participant to connect a series of 25 circles numbered 1-13 randomly interspersed with letters $\mathrm{A}-\mathrm{L}$, alternating between numbers and letters ( 1 to $\mathrm{A}, \mathrm{A}$ to 2 , 2 to $\mathrm{B}$, etc.). The investigator points out errors as they occur so that the participant can make the corrections. For both Part A and Part B, the score is the number of seconds required to complete the test, including the time for corrections.

Three Shapes (TS) and Three Words (TW) (Mesulam, 1985) are measures of the effectiveness of short-term memory and retention of information including acquisition of new information, which require attentional control. The participant is asked to copy six test stimuli, i.e. three shapes and three words with low imagery value. One minute after the stimuli are removed the individual is asked to reproduce them from memory. The scores are the number of correct responses in reproducing the words and the shapes in immediate recall.

Attentional Functional Index (AFI) (Cimprich, 1992) was used to measure subjective or perceived effectiveness in cognitive activities requiring directed attention such as planning daily activities, getting started on tasks, making decisions, keeping a train of thought, remembering to do important things, and attending to details. The instrument consists of 16 linear analogue scales (each $100 \mathrm{mms}$.) labeled at either end with polar opposite phrases (e.g. 'not at all' and 'extremely well'). The respondents were asked to place a mark through the horizontal line at whatever point best described how well they were functioning at the time. Validity and reliability have been established in healthy and ill populations (Cimprich, 1992, 1999). In this sample, the internal consistency coefficient was 0.91 , indicating good reliability.

Symptom Distress Scale (SDS) (McCorkle and Young, 1978) was used to measure the degree of distress experienced in relation to 10 common symptoms such as fatigue, insomnia, and loss of appetite. Responses were made on a five-point scale ranging from 1 (no problem) to 5 (the worst possible problem). The SDS has been widely used in studies involving patients with cancer and has established validity and reliability (McCorkle and
Quint-Benoliel, 1983). In this research, the internal consistency coefficient was 0.80 , indicating satisfactory reliability.

Profile of Mood States-Short Form (POMS-SF) (McNair et al., 1992) was used to assess mood state. The short form consists of 30 adjectives that represent six mood states, namely, anxiety, anger, depression, vigor, confusion, and fatigue. Respondents rated themselves on a five-point scale ranging from 0 (not at all) to 4 (extremely) in response to feelings during the past week. Because of scoring procedures with vigor negatively weighted, the summed score on the measure can range from a low of -20 (no distress) to high of 100 (worst distress). Validity and reliability of the POMS and subscales have been established in varying populations. In this research, internal consistency for the subscales ranged from 0.78 to 0.92 and the coefficient for the total POMS-SF was 0.87 .

\section{PROCEDURE}

The university's Health Sciences Institutional Review Board approved the study. Following written consent, testing was conducted during a scheduled pre-treatment clinic visit in a quiet, private room in the ambulatory care area. A trained research assistant administered the tests in random sequence using standard formats and instructions. Testing occurred at about 23 days following diagnosis by biopsy and about 18 days before surgery.

\section{DATA ANALYSIS}

Descriptive statistics were used to characterize the sample of women newly diagnosed with breast cancer on demographic and medical variables. Pearson correlation coefficients were used to determine relationships between cognitive variables and selected demographic and distress variables. Multiple regression analyses were performed to determine possible predictors of cognitive functioning prior to breast cancer treatment. For certain analyses, a composite score of attentional performance, the Total Attentional Score (TAS), was computed by transforming raw scores on the objective tests to $\mathrm{Z}$ scores based on the mean and standard deviation of the sample, 
reversing scores on certain tests so that higher scores represented better performance, and summing the standardized scores (DSF + DSB + $\mathrm{TMA}+\mathrm{TMB})$. For all other analyses, raw scores were used.

\section{RESULTS}

\section{Performance on cognitive measures}

Scores on the objective tests of attention and short-term memory are reported in Table 2. When compared with available age-normed data (Lezak, 1995; Spreen and Strauss, 1998), the mean scores of the attention tests generally fell within the normal range for healthy adults. On the subjective measure of perceived effectiveness in cognitive functioning, the AFI, scores ranged from a low of 17 to a high of 97 with a mean of $65.7(\mathrm{SEM}=1.38)$. A further examination of the frequencies indicated that only $25 \%$ scored above 75 indicating effective functioning, with $50 \%$ reporting moderate effectiveness (score of 50-75) and $25 \%$ reporting lower effectiveness (score below 50).

Relationship of cognitive measures: age, menopausal status, education, and comorbidity

The correlations between scores on cognitive measures and selected study variables are shown in Table 3. There were no significant relationships between scores on the self-report measure, the AFI, and performance on the objective tests of attention and short-term memory.

Age was significantly correlated with the objective attention and short-term memory measures with the exception of DSF. The Pearson correlations were small to moderate ranging from 0.16 to 0.47 with older age related to poorer

Table 2. Test scores of total sample

\begin{tabular}{lccc}
\hline & $M$ & SEM & Min-Max \\
\hline Attention tests: & & & 0.10 \\
$\quad$ Digit Span Forward (DSF) & 6.62 & 0.10 & $4-9$ \\
Digit Span Backward (DSB) & 4.75 & 1.24 & $3-8$ \\
Trail Making Test A (TMA) (s) & 34.18 & 2.70 & $13-119$ \\
Trail Making Test B (TMB) (s) & 71.33 & & $21-285$ \\
Short-term memory: & & 0.04 & $0-3$ \\
Three Shapes (1 min) & 2.80 & 0.05 & $0-3$ \\
Three Words (1 min) & 2.61 & 1.38 & $17-97$ \\
Rating scales: & & 0.43 & $11-36$ \\
Attentional Functional Index (AFI) & 65.69 & 1.54 & $-13-82$ \\
Symptom Distress Scale (SDS) & 20.51 & & \\
Profile of Mood State (POMS-SF) & 19.40 & & \\
\hline
\end{tabular}

Table 3. Correlation of cognitive measures with selected demographic and distress variables

\begin{tabular}{|c|c|c|c|c|c|}
\hline & AFI & Age & Education & POMS-SF & SDS \\
\hline Digit span forward & -0.04 & -0.12 & $0.22 *$ & 0.12 & 0.10 \\
\hline Digit span backward & -0.11 & $-0.18^{*}$ & $0.27^{* * *}$ & $0.18^{*}$ & 0.11 \\
\hline Trail making A & 0.04 & $0.47^{* * *}$ & -0.13 & -0.11 & -0.03 \\
\hline Trail making B & 0.02 & $0.44^{* * *}$ & $-0.22^{* *}$ & $-0.21^{* *}$ & $-0.17^{*}$ \\
\hline Total attention score & -0.12 & $-0.43^{* * *}$ & $0.29^{* * *}$ & $0.23^{*}$ & 0.15 \\
\hline Three shapes ( $1 \mathrm{~min})$ & -0.07 & $-0.16^{*}$ & $0.18^{*}$ & 0.12 & -0.06 \\
\hline Three words (1 min) & -0.01 & $-0.16^{*}$ & 0.02 & 0.04 & -0.13 \\
\hline Attentional function index & - & $0.23^{* *}$ & 0.01 & $-0.62 * * *$ & $-0.58 * * *$ \\
\hline
\end{tabular}

Note: AFI = Attentional Function Index; POMS-SF $=$ Profile of Mood States-Short Form; SDS = Symptom Distress Scale.

${ }^{*}<0.05 ;{ }^{* *}<0.01 ; * * * 0.001$. 
performance. Age also was significantly related to scores on the subjective measure, the AFI; however, in contrast to performance on objective measures, younger age was associated with perceptions of poorer effectiveness in cognitive functioning.

Menopause status groups (pre-, peri-, and postmenopausal) differed significantly on three tests (not tabled), TMA and TMB and the Three Words Recall, with the post-menopausal (older) group having poorer performance, $p<0.05$, on these tests as compared to the pre-menopausal (younger) group (TMA: $M=27$ versus 37; TMB: $M=53$ versus 81 ; Three Words Recall: $M=2.8$ versus 2.5 , pre- and post-menopausal groups, respectively). The small group of peri-menopausal women $(n=13)$ did not differ significantly from either the pre- or post-menopausal groups on any of the objective measures.

On the subjective measure of attentional functioning, the AFI, there was a significant difference between the pre- and post-menopausal groups, with the pre-menopausal (younger) group reporting significantly lower effectiveness in cognitive functioning $(M=60), F(2171)=3.19, \quad p=0.04$, compared to the post-menopausal (older) group $(M=68)$. Again, the peri-menopausal group did not differ $(M=65)$ from either the preor post-menopausal groups on this subjective measure.

Years of education were significantly correlated with the objective measures with the exceptions of Trail Making A and the Three Words memory task. The size of the correlations in this relatively well-educated sample was small, ranging from 0.18 to 0.29 , and generally indicated that more years of education were associated with better performance on these cognitive measures. There was no significant relationship between years of education and scores on the AFI, the subjective measure of cognitive functioning.

Presence of other chronic health problems was associated with significant differences in performance on the cognitive measures, with the group reporting presence of chronic health problems having significantly poorer scores on TMA, $t(177)=-4.57, p<0.001$, TMB, $t(177)=-4.37$, $p<0.001$, and TW $(1 \mathrm{~min}), t(177)=2.93, p=0.004$. There were no significant differences in mean scores on the subjective measure of cognitive functioning, the AFI, based on presence or absence of a chronic health problem.
Relationship of cognitive measures and symptom distress

The scores on the symptom distress scale ranged from a low of 11 to a high of 36 with a mean of $20.51(\mathrm{SEM}=0.43)$ indicating a low to moderate level of symptom distress prior to any treatment (see Table 2). The most frequently reported symptoms that produced some level of distress were mood state disturbance $(91 \%)$, insomnia $(76 \%)$, and fatigue $(75 \%)$. There were no significant relationships between symptom distress scores and performance on the objective measures of attention and short-term memory with the exception of one test (TMB) (see Table 3). However, there was a significant, moderately strong, inverse relationship between symptom distress and scores on the AFI. As symptom distress increased, perceived effectiveness in cognitive functioning decreased, and vice versa.

The scores on the POMS-SF, a measure of affective distress, ranged from a low of -13 to a high of 82 (see Table 2). Given the possible range of scores, the mean of $19.40(\mathrm{SEM}=1.54)$ indicated an overall low level of mood distress prior to treatment. At the same time, $25 \%$ of the participants scored over 30 on the measure, indicating presence of moderate to high distress. There were small but significant relationships $(p<0.05)$ between POMS-SF and scores on two cognitive measures, DSB and TMB (see Table 3). Of interest, the direction of the correlations indicated that better performance on these two measures was associated with higher affective distress. A further examination of relationships of cognitive measures with POMS-SF subscale scores indicated that, in particular, anxiety scores were inversely correlated with performance of the cognitive measures suggesting that increased anxiety tended to enhance performance on these tests.

There was a significant and strong relationship between POMS-SF scores and the subjective measure of effectiveness in cognitive function, the AFI. Higher levels of perceived effectiveness in cognitive functioning were associated with lower levels of affective distress.

\section{Pre-treatment predictors of cognitive functioning}

To determine possible predictors of performance on objective measures of directed attention in the pre-treatment period, a multiple regression 
Table 4. Multiple regression analysis of Total Attention Score (TAS) ${ }^{\mathrm{a}}$ to identify pre-treatment predictors

\begin{tabular}{lrrrr}
\hline Variables & \multicolumn{1}{c}{$B$} & SE $B$ & Beta & Significance \\
\hline Age & -0.11 & 0.03 & -0.43 & $<0.001$ \\
Education & 0.33 & 0.08 & 0.28 & $<0.001$ \\
Health problems & -0.51 & 0.41 & -0.09 & 0.21 \\
Profile of mood states & 0.02 & 0.01 & 0.18 & 0.07 \\
Symptom distress & -0.01 & 0.05 & -0.03 & 0.78 \\
Menopausal status & 0.80 & 0.59 & 0.14 & 0.18 \\
\hline
\end{tabular}

${ }^{\mathrm{a}} \mathrm{TAS}=$ Total Attentional Score (sum of standardized scores DSF + DSB + TMA + TMB).

Table 5. Multiple regression analysis of Attentional function Index (AFI) to identify pre-treatment predictors

\begin{tabular}{lcccc}
\hline Variables & $B$ & SE $B$ & Beta & Significance \\
\hline Age & -0.006 & 0.14 & -0.004 & 0.96 \\
Education & 0.38 & 0.45 & 0.05 & 0.41 \\
Health problems & 0.14 & 2.25 & 0.004 & 0.95 \\
Menopausal status & 1.79 & 1.74 & 0.09 & 0.30 \\
Symptom distress & -1.02 & 0.26 & -0.32 & $<0.001$ \\
Profile of mood states & -0.34 & 0.07 & -0.38 & $<0.001$ \\
\hline
\end{tabular}

analysis was performed using the composite Total Attention Score (TAS) as the dependent variable. Age, years of education, menopausal status (postversus pre- or peri-menopausal), presence or absence of chronic health problems, and overall scores for Symptom Distress Scale (SDS) and POMS-SF were entered as independent variables. Together these variables accounted for $29 \%$ of the variance in the TAS prior to breast cancer treatment, Multiple $R=0.54, F(6,162)=10.48$, $p<0.001$ (see Table 4). Only two variables, age and years of education, were found to be significant predictors of objective attentional performance. Conversely, menopausal status, presence of a chronic health problem, and levels of symptom (SDS) and affective (POMS-SF) distress were not significant predictors of attention in the pre-treatment period.

To determine possible predictors of subjective or perceived effectiveness in cognitive functioning in women prior to treatment for breast cancer, a multiple regression analysis was performed using the overall AFI score as the dependent variable. Again, age, years of education, menopausal status, other chronic health problems, and overall scores for SDS and POMS-SF were entered as independent variables as described above. Together these variables explained $43 \%$ of the variance in the AFI scores, Multiple $R=0.65, F(6,168)=20.14$, $p<0.001$ (see Table 5). Two variables, SDS and POMS-SF scores, were found to be significant predictors of perceived effectiveness in cognitive functioning, whereas age, education, menopausal status and presence of chronic health problems were not significant predictors.

\section{DISCUSSION}

This study examined individual factors associated with the cognitive capacity to direct attention prior to any treatment in women newly diagnosed with breast cancer. The findings indicated that scores on the objective measures were not significantly related to subjective perceptions of cognitive functioning in activities requiring directed attention. Furthermore, when controlling for possible covariates, age and education were significant predictors of overall performance on the objective measures of attention prior to any treatment, while levels of symptom and affective distress significantly predicted perceptions of the effectiveness in cognitive functioning.

As might be expected, older age was associated with poorer performance on the measures. In contrast, on the self-report measure, younger women reported less effectiveness in cognitive 
functioning than the older women, suggesting that younger women may have perceived even small fatigue-related losses in attention that interfered with usual levels of functioning, but that were not detectable on testing. Older women, however, may have adapted their expectations of effective functioning in keeping with life demands and a more gradual decline in attentional performance over time. Despite reports of more effective functioning, however, older women demonstrated decreased ability to direct attention prior to any treatment, indicating a greater susceptibility to possible treatment-related losses in cognitive functioning over time.

Higher educational level was related to better performance on most of the objective measures, although the relationships were relatively small. The sample tended to be well educated, however, and this may have accounted for the small size of the observed relationships. Nevertheless, education did remain a significant predictor of attentional performance even after taking into account age and other possible covariates.

Menopausal status was not a significant predictor of overall performance on the attention measures when taking into account age, suggesting that age may explain the observed relationship of menopausal status with cognitive functioning. Also, although presence of a chronic health problem, such as diabetes, hypertension, and heart disease, was associated with poorer performance on objective measures, having a co-morbid condition was not a significant predictor of attentional performance when controlling for other covariates. Thus, the study provides clear evidence of the effect of age that is not explained by menopausal status or chronic illness, but no evidence of the effects of menopausal status or chronic illness that is not explained by age.

Distress levels generally were not associated with performance on the objective tests. The mean scores on the POMS-SF indicated generally low to moderate levels of distress prior to the start of treatment (about three weeks following diagnosis). It is possible that such low to moderate levels of distress were not sufficient to have any effect on performance of the selected tests, and that moderately heightened anxiety could enhance attention.

In relation to subjective perceptions of attentional functioning, a different pattern of relationships and predictors emerged. Perceptions of cognitive problems were related to higher psycho- logical distress but not to actual performance on the objective tests of attention and short-term memory. After controlling for possible covariates, symptom distress and affective distress were significant predictors of how well the women perceived they were functioning in everyday activities that required directed attention such as carrying out effortful tasks, making decisions, and being able to take in information. Similar results have been reported in samples of lymphoma patients in remission (Cull et al., 1996) and of breast cancer patients following standard or high dose chemotherapy (van Dam et al., 1998). In those studies, complaints of problems in attention and memory were related to greater affective distress but not to performance on objective measures. Cull et al. (1996) proposed that complaints of problems in attention and memory reflected affective disorder and mental fatigue. An explanation of affective disorder seems unlikely in this sample since scores on the POMS-SF generally reflected low to moderate levels of distress. One other explanation may be that even small fatigue-related losses in attention may contribute to overall experience of distress because keeping up with the demands of daily life becomes more difficult. Because the neuropsychological tests used in this study do not reflect the complexities of daily tasks, they may be less sensitive to subtle losses in cognitive functioning that might be perceived by an individual. In this regard, women in this study frequently described distressing lapses in attention and memory that occurred since the diagnosis of breast cancer. Such losses in cognitive function following prolonged or intense mental demands have been associated with mental or 'attentional' fatigue (Kaplan, 1995) and appear to be amenable to interventions to enhance attention (Cimprich, 1993; Cimprich and Ronis, 2003).

Overall the findings provide new evidence of factors that might influence attentional functioning even prior to breast cancer treatment. Of interest, the findings suggest that self-reports are not accurate indicators of cognitive performance. At the same time, measured performance on tests such as those used in this study may not accurately reflect the cognitive problems that many women experience following diagnosis of breast cancer. Importantly, the findings indicate that different factors were associated with measured performance versus self-reports of cognitive functioning. Longitudinal investigations are especially needed to better understand how individual factors such 
as age and distress may predispose individuals to cognitive impairments, particularly losses in attention, during and following breast cancer treatment.

\section{ACKNOWLEDGEMENTS}

The authors thank Barbara Therrien for her comments during the preparation of this article. Supported by the National Institutes of Health, National Institute of Nursing Research, R29 NR04132.

\section{REFERENCES}

Ahles TA, Saykin A. 2001. Cognitive effects of standarddose chemotherapy in patients with cancer. Cancer Invest 19(8): 812-820.

Allen PA, Hall RJ, Druley JA, Smith AF, Sanders RE, Murphy MD. 2001. How shared are age-related influences on cognitive and neurocognitive variables? Psychol Aging 16(3): 532-539.

Bender CM, Paraska KK, Sereika SM, Ryan CM, Berga SL. 2001. Cognitive function and reproductive hormones in adjuvant therapy for breast cancer. $J$ Pain Symptom Manage 21(5): 407-424.

Braver TS, Barch DM, Keys BA et al. 2001. Context processing in older adults: Evidence for a theory relating cognitive control to neurobiology in healthy aging. J Exp Psych: Gen 130(4): 746-763.

Capitani E, Barbarotto R, Laiacona M. 1996. Does education influence the age-related cognitive decline? A further inquiry. Dev Neuropsychol 12: 231-240.

Cimprich B. 1992. Attentional fatigue following breast cancer surgery. Res Nurs Health 15: 199-207.

Cimprich B. 1993. Development of an intervention to restore attention in cancer patients. Cancer Nurs 16(2): 83-92.

Cimprich B. 1999. Pretreatment symptom distress in women newly diagnosed with breast cancer. Cancer Nurs 22(3): 185-194.

Cimprich B, Ronis D. 2001. Attention and symptom distress in women with and without breast cancer. Nurs Res 50(2): 86-94.

Cimprich B, Ronis D. 2003. An environmental intervention to restore attention in women with newly diagnosed breast cancer. Cancer Nurs 26(4): 284-292.

Cull A, Hay C, Love SB, Mackie M, Smets E, Stewart M. 1996. What do cancer patients mean when they complain of concentration and memory problems? Br J Cancer 74: 1674-1679.

James W. 1983. The Principles of Psychology. Harvard University Press: Cambridge, MA. (original work published 1890).
Kaplan S. 1995. The restorative benefits of nature: Toward an integrative framework. J Environ Psychol 15: $169-182$.

Kaplan S, Kaplan R. 1982. Environment and Cognition. Praeger: New York.

Leckliter IN, Matarazzo JD. 1989. The influence of age, education, IQ, gender, and alcohol abuse on Halstead-Reitan Neuropsychological Test Battery performance. J Clin Psychol 45: 484-512.

Lezak MD. 1995. Neuropsychological Assessment. Oxford University Press: New York.

McCorkle R, Quint-Benoliel J. 1983. Symptom distress, current concerns and mood disturbance after diagnosis of life-threatening disease. Soc Sci Med 17: 431-438.

McCorkle R, Young K. 1978. Development of a symptom distress scale. Cancer Nurs 1: 373-378.

McNair DM, Lorr M, Droppleman LF. 1992. EDITS Manual for the Profile of Mood States. EDITS/ Educational and Industrial Testing Service: San Diego, CA.

Mesulam MM. 1985. Principles of Behavioral Neurology. FA Davis: Philadelphia.

Phillips K-A, Bernhard J. 2003. Adjuvant breast cancer treatment and cognitive function: Current knowledge and research directions. J Nat Cancer Inst 95(3): 190-197.

Posner MI. 1995. Attention in cognitive neuroscience: An overview. In The Cognitive Neurosciences, Gazzaniga MS (ed.). MIT Press: Cambridge MA; 615-624.

Posner MI, Dehaene S. 1994. Attentional networks. Trends Neurosci 17(2): 75-79.

Reitan RM. 1958. Validity of the trail-making test as an indicator of organic brain damage. Percept Mot Skills 8: 271-276.

Smith EE, and Jonides J. 1999. Storage and executive processes in the frontal lobes. Science 283: 1657-1661.

Spreen O, Strauss E. 1998. A Compendium of Neuropsychological Tests: Administration, Norms and Commentary. Oxford University Press: New York; 533-547.

van Dam F, Schagen SB, Muller MJ et al. 1998. Impairment of cognitive function in women receiving adjuvant treatment for high-risk breast cancer: High dose versus standard-dose chemotherapy. $J$ Nat Cancer Inst 90(3): 210-218.

Ylikoski R, Ylikoski A, Erkinjuntti T et al. 1998. Differences in neuropsychological functioning associated with age, education, neurological status, and magnetic resonance imaging findings in neurologically healthy elderly individuals. Appl Neuropsychol 5: $1-14$.

Zacks R, Hasher L. 1994. Directed ignoring: Inhibitory regulation of working memory. In Inhibitory Processes in Attention, Memory and Language. Dachenbach D, Carr TH (eds). Academic: San Diego, CA; 241-264. 